\title{
Educación y desarrollo en Brasil, 1995-2000
}

\section{Paulo Renato Souza}

Ministro de Educación de la República Federativa de Brasil

paulorenato@gm.mec.gov.br
Este artículo analiza las políticas de educación del sexenio 1995-2000 en el Brasil. Tras señalar la necesidad de preparar al ciudadano y al país para enfrentar el siglo XXI, considera el atraso secular de la educación brasileña y las características generales del sistema educativo en dicho país. Enseguida describe las opciones de la política educacional del período, que apuntan sobre todo a la expansión con calidad del sistema; analiza las particularidades de los programas para la educación básica (entendida como la educación que se imparte desde los cero años hasta el fin de la enseñanza media); los programas compensatorios orientados a la retención escolar; la educación especial; los planes de alfabetización y la educación de jóvenes y adultos. Examina la formación de docentes, la enseñanza secundaria y técnico-profesional y la educación superior. Considera temas relativos a la transparencia de la información sobre el sistema educativo y a las posibilidades de evaluarlo. Pasa revista asimismo al financiamiento de la educación y la puesta en práctica de las normas constitucionales correspondientes. El artículo concluye con la consideración de los desafíos y perspectivas de la educación en Brasil, señalando como reto principal la búsqueda de índices de calidad crecientes en todos los niveles educativos, lo que está íntimamente ligado a la calificación del cuerpo docente y al financiamiento del sistema. 


\section{I \\ Preparando el ciudadano y el país del siglo XXI}

Con una población de 165 millones de habitantes, Brasil es el quinto país más populoso del planeta, sólo superado por China, India, Rusia y Estados Unidos. Es el cuarto en extensión territorial y está entre las diez mayores economías del mundo.

Su sociedad y economía son diversificadas y complejas, con enormes potencialidades pero también con graves problemas, herencia del pasado. Existe hoy en el país una clara conciencia de que invertir en educación es la vía principal para enfrentar esos problemas $\mathrm{y}$ transformar en realidad la enorme esperanza con que su pueblo encara el futuro.

Consciente de la importancia estratégica de la educación para insertar el país en una economía global cada vez más anclada en el conocimiento y la innovación, Brasil pasó a considerar la enseñanza como una prioridad nacional. Además de tomar medidas destinadas a garantizar el financiamiento del sector, durante los últimos seis años aplicó en forma continua un conjunto coherente de políticas en todos los niveles de la educación brasileña.

Como telón de fondo de esas políticas está la comprensión de que el mundo vive un gran proceso de cambio estructural, desde el punto de vista de su historia económica, con todas las consecuencias sociales y políticas que esto significa. El modo de producir dio un salto gigantesco, lo que tuvo un enorme efecto sobre el consumo, el empleo, los salarios y, especialmente, sobre la productividad del trabajo.
El surgimiento de la informática revolucionó la tecnología de producción industrial, tornando obsoletas las antiguas especializaciones y requiriendo un nuevo tipo de trabajador, más versátil y mejor equipado intelectualmente. El gran salto de las comunicaciones, potenciado por el desarrollo de la tecnología espacial, rompió literalmente las barreras nacionales: a los países se les hizo imposible mantenerse aislados en un mundo con acceso instantáneo a la información, y se tornó inviable la aparición de procesos de desarrollo nacionales autóctonos.

En este mundo globalizado e interdependiente en una escala nunca antes vista en la historia universal, la superación de la pobreza y el arraigamiento de la democracia pasan necesariamente por la capacidad de apropiación y generación del conocimiento científico y tecnológico, raíz y motor del dinamismo del capitalismo contemporáneo, y por la afirmación de una ciudadanía dotada de los instrumentos para comprender, criticar e influir en la definición de los rumbos de dichas transformaciones.

Para la educación, el desafío es doble: formar el individuo y preparar el país tanto para comprender un mundo nuevo cuyas bases se reorganizan por completo como para participar en él. Recuperar el tiempo perdido, superar las deficiencias básicas y esenciales heredadas del pasado y, al mismo tiempo, implantar la reforma educacional que exige la nueva sociedad, es una tarea gigantesca que exige de los gobiernos y la sociedad la asignación de una clara prioridad a la educación.

\section{II}

\section{Un atraso secular en la educación brasileña}

Pese a todas las deficiencias y desigualdades de su modelo de desarrollo elitista y concentrador, Brasil ocupó el segundo lugar entre los países con mayor crecimiento económico del mundo entre 1890 y 1980. Pero fue el último país en abolir la esclavitud, en 1889, y el modelo agrario brasileño siguió manteniendo en la exclusión y la ignorancia a las grandes masas cam- pesinas, descendientes en gran medida de los esclavos y de poblaciones indígenas que han perdido sus características culturales.

El proceso de industrialización —entre 1930 y 1980 - se caracterizó por la sustitución de importaciones, sin hacer hincapié en la competitividad o la innovación. Las exportaciones se originaban sólo en los 
sectores que hacían uso intensivo de recursos naturales y mano de obra barata, y la alta concentración del ingreso convivía con la profundización de la exclusión social.

Hasta 1950 , casi $70 \%$ de la población todavía vivía en el campo. En 1960, sólo 60\% de los niños de siete a catorce años asistía a la escuela y la tasa de analfabetismo era de 40\%. Pero ya en 1997 el $80 \%$ de los brasileños vivía en las ciudades. Ese vertiginoso proceso de urbanización estuvo acompañado hasta fines de los años ochenta por altas tasas de crecimiento demográfico. Las grandes metrópolis, especialmente, tuvieron que acoger en sus redes ya deficientes de equipamiento y servicios urbanos a una población joven de origen rural, desprovista de casi toda calificación para las actividades productivas de la economía industrial.

A mediados de los años noventa, 89\% de los niños asistía a la escuela y la tasa de analfabetismo había caído a $16 \%$. Eran avances considerables, aunque insuficientes ante las exigencias de la nueva sociedad del conocimiento, sobre todo si se considera que sólo $50 \%$ de los niños que iniciaban el primer curso primario terminaba los ocho cursos, tardando un promedio de doce años en completar el ciclo, debido a las altísimas tasas de repitencia y deserción escolar.
Este panorama educacional desolador —el país tiene todavía hoy un legado de 15 millones de analfabetos mayores de 14 años - era coherente y hasta "funcional' frente al modelo de desarrollo vigente hasta entonces, que se hallaba al servicio de una política proteccionista y era capaz de equilibrarse internamente, protegido de la competencia internacional. Para esta modalidad de desarrollo industrial privilegiada por las elites, bastaba con tener en la educación un segmento de buena calidad que abarcara todos los niveles (primario, secundario y universitario), sin preocuparse por ampliar la cobertura, pues no era prioritario que toda la población recibiese educación.

Dentro de estos límites, el país siguió ese modelo educacional con cierta competencia, ya que en él se desarrolló y prosperó, sobre todo entre los años sesenta y ochenta, la mejor especialización de posgrado de los países en desarrollo.

Pero, sobre todo a partir de los años noventa, se intensificó la presión por lograr mejores condiciones educacionales para la población en general, ejercida tanto por los jóvenes y las familias que buscaban el ascenso económico y social como por la propia economía, cada vez más urbanizada y compleja, y cada vez más expuesta a la competencia externa.

\section{III}

\section{Características del sistema de educación brasileño}

Una particularidad del sistema educacional brasileño es su carácter extremadamente diversificado y descentralizado. El país es una república federal, constituida por 26 estados federativos y el distrito federal. Las unidades de la federación se subdividen a su vez en municipios, los que actualmente bordean los seis mil.

Todo el sistema de educación, excepto el de nivel superior, es predominantemente público. Existen hoy en el país, matriculados en todos los niveles de enseñanza, 54.7 millones de estudiantes. Esta cifra representa cerca de un tercio de la población general y equivale prácticamente a la suma de las poblaciones totales de Argentina, Chile y Uruguay. Las escuelas del sector público dan educación gratuita al $89 \%$ del total de 52 millones de alumnos de educación básica —in- fantil, primaria y media - que equivalen a la suma de las poblaciones totales de Venezuela y Perú.

La nueva Ley de Directrices y Bases de la Educación Nacional, promulgada en diciembre de 1996, redefinió los papeles y responsabilidades de cada sistema de enseñanza (federal, estadual y municipal). Al dar mayor autonomía a la escuela, flexibilizar los contenidos curriculares y estimular la calificación del magisterio - estipula que para el año 2007 todos los profesores de educación básica deben tener formación superior-, la nueva ley creó el ambiente necesario para poner en marcha cambios significativos en el escenario educacional del país.

La educación primaria (fundamental) obligatoria que abarca de primero a octavo grado, así como 
las guarderías infantiles (cero a tres años), la enseñanza preescolar (cuatro a seis años) y la educación media han sido siempre responsabilidad de estados y municipios. El papel del gobierno central en esos niveles es normativo, pues establece los grandes lineamientos del sistema redistributivo y subsidiario, otorgando asistencia y subsidios para disminuir las desigualdades sociales y regionales. Sólo las instituciones de enseñanza superior y las escuelas técnico-profesionales de nivel medio son mantenidas directamente por la Unión.

A partir de la aprobación de la Ley de Directrices y Bases de la Educación Nacional se desencadenó un amplio proceso de "municipalización" de la educación primaria y de "estadualización" de la educación media. En 1997 había 18 millones de alumnos en las escuelas estaduales y 12 millones en las municipales. En 1999, las redes municipales y estaduales se igualaron con 16 millones de alumnos cada una. La enseñanza media, que se expandió a tasas medias de $14 \%$ entre 1996 y 1999, se ha concentrado cada vez más en las redes estaduales. Los datos preliminares del censo escolar de 2000 muestran que la expansión de 5.4\% de la matrícula se originó en la red estadual, que creció $7.9 \%$, mientras que todas las demás redes experimentaron descensos: $-10.2 \%$ la federal, $-4.9 \%$ la municipal y $-4.2 \%$ la privada.

Este movimiento es coherente con la Ley de Directrices y Bases de la Educación Nacional, que prevé la oferta de educación media por los estados.

\section{IV}

\section{Las grandes opciones de la nueva política educacional del gobierno federal}

Todo el esfuerzo del gobierno federal se concentra actualmente en promover, de la base a la cima de la estructura, la expansión con calidad de las oportunidades educacionales; está dirigido especialmente a la población en edad escolar, pero busca asegurar también oportunidades de ingreso a quienes no tuvieron acceso a la educación en edad adecuada, tomando en cuenta el derecho de los brasileños en general a la ciudadanía y el modelo de desarrollo necesario para que esa ciudadanía pueda ejercerse plenamente.

En la enseñanza primaria, lo más importante era matricular y mantener a los niños de 7 a 14 años en la escuela, garantizándoles una enseñanza de calidad. El segundo objetivo del gobierno, después del de universalizar el acceso, fue el de asegurar el éxito escolar, representado por el avance de los alumnos hasta la conclusión del último curso dentro del tiempo previsto, meta que incluía necesariamente la mejoría de la calidad de la educación.

Pero también cabe destacar, junto a la prioridad otorgada a la cobertura universal de la enseñanza primaria, las medidas relativas a la reforma y diversificación de la enseñanza media y técnico-profesional correspondiente, a la formación y capacitación de los profesores y a la expansión y evaluación de la enseñanza superior.
Los aspectos cuantitativos y cualitativos de esas políticas fueron sometidos a un control permanente a través de mecanismos confiables de información y evaluación educacional, de manera que, habiendo partido de cero hace cinco años, hoy se cuenta con un sistema que puede compararse con los de los países más desarrollados.

Además, se han elaborado programas complementarios de carácter compensatorio - algunos de los cuales se incluyen entre los más integrales del mundodestinados a contrarrestar una herencia histórica de desigualdades en la sociedad brasileña. Entre ellos cabe destacar la distribución gratuita de la merienda escolar y el libro didáctico en la enseñanza primaria, la complementación del ingreso de las familias más pobres que tienen niños en la escuela y el crédito educativo para los jóvenes que llegan a la educación superior. Además, los programas de aceleración del aprendizaje, de alfabetización y educación de jóvenes y adultos y de apoyo a los sistemas educacionales de las regiones Norte y Nordeste también están orientados a compensar desequilibrios regionales y de ingreso, y a contribuir al gran esfuerzo de inclusión social hoy en marcha en Brasil.

El programa educacional del gobierno, basado en la búsqueda de la igualdad de oportunidades, incorpo- 
ró junto a los principios de cobertura universal, calidad y descentralización, la participación de la comunidad en la gestión de las escuelas y el mayor control social del gasto público y sus resultados. Para ello, la tradicional asignación de recursos federales a los entes federados mediante convenios negociados fue sustituida gradualmente por mecanismos de transferencia automática basados en criterios transparentes y universales. Los instrumentos burocráticos de seguimiento y evaluación fueron sustituidos por la participación de la comunidad en órganos colegiados de supervisión y fiscalización.

\section{Educación primaria (fundamental)}

En cinco años se registró una fuerte expansión del acceso a la enseñanza primaria. La proporción de niños de 7 a 14 años matriculados en las escuelas aumentó de $89 \%$ en 1994 a $96 \%$ en 1999, habiéndose incorporado al sistema 4 millones de alumnos en ese período. Las desigualdades regionales están disminuyendo. En la región Nordeste, la matrícula creció casi $27 \%$, comparada con $13 \%$ para el país en su conjunto.

$\mathrm{Al}$ contrario de lo que suele suponerse, las escuelas públicas de la enseñanza básica tendrían vacantes suficientes - naturalmente, con adaptaciones a un perfil diferente de estudiantes - para absorber a toda la población de 7 a 14 años (correspondiente a la educación primaria obligatoria) y de 15 a 17 años (correspondiente a la enseñanza media). Según el censo escolar de 1999, Brasil tenía 44 millones de alumnos matriculados en la educación básica pública, mientras que la población de 7 a 17 años era de 37 millones. El exceso de alumnos proviene de la repitencia, altísima en los primeros grados, y del ingreso tardío.

Pese a que la distorsión edad/grado sigue siendo alta $-46.6 \%$ de los estudiantes podrían ir más adelantados- Brasil está mejorando su desempeño en la educación primaria.

Entre 1994 y 1999, el número de egresados de la enseñanza primaria saltó de 1588000 a 2383 000, lo que representa un crecimiento de $50.1 \%$. En el mismo período, el número de egresados de la enseñanza media aumentó en $67.8 \%$, es decir, de 915000 a 1535000 .

La tasa de promoción, que mide el número de alumnos que pasan de curso, aumentó de 65\% en 1995 a 74\% en 1998; la expectativa de conclusión del primer grado se elevó a $63 \%$ y el tiempo medio para terminar los ocho grados se redujo de 12 a 10 años. En el año 2000 comenzó a disminuir por primera vez la matrícula en el ciclo primario, especialmente en los primeros cuatro grados, ya que la del quinto al octavo grado sigue creciendo. Hay más alumnos concluyendo la enseñanza primaria que ingresando a ella, lo que significa que la distorsión edad/grado y los índices de reprobación están disminuyendo. Esta mayor eficiencia alivia las cargas educacionales de los municipios y amplía el desafío de los estados responsables de velar por la expansión de la enseñanza media.

Un elemento importante de la recuperación del desfase edad/grado es el programa de aceleración del aprendizaje. Por su intermedio, el gobierno federal financia el establecimiento de clases especiales para alumnos con alto desfase en este sentido, para procurar que avancen rápidamente hasta el grado compatible con su edad. Entre 1998 y 2000 más de 3.5 millones de alumnos se beneficiaron del programa, consiguiendo la mayoría de ellos progresar en sus estudios.

Se reitera que el principal problema de la enseñanza primaria no es cuantitativo, sino cualitativo. La formulación y difusión de los parámetros y referencias curriculares nacionales para todos los niveles y modalidades de la enseñanza básica (educación preescolar, primaria, media, de jóvenes y adultos e indígena), y también programas como TV Escola o Proinfo, que se tratarán más adelante, se suman al mejoramiento y extensión de otros programas, como el del libro didáctico y el de bibliotecas escolares, para responder al desafío de elevar la calidad de la educación primaria.

A los profesores primarios se les hizo entrega de más de 1.4 millones de ejemplares de los parámetros y referencias curriculares. En 1998, se distribuyeron a las escuelas 20 mil colecciones de la llamada biblioteca del profesor, compuesta por obras de referencia sobre la formación histórica, social y política de Brasil, y en 1999 se entregaron otras 35 mil bibliotecas de literatura infantil y juvenil destinadas a alumnos de la enseñanza primaria.

Los programas de carácter compensatorio, orientados a corregir los efectos de las grandes disparidades sociales y de ingreso que afectan a gran parte de las familias brasileñas, contribuyen también a aumentar las condiciones propicias para el éxito escolar de los estudiantes de bajos ingresos.

\section{Programas compensatorios}

El Programa Nacional de Alimentación Escolar (PNAE), conocido como "merienda escolar", proporciona durante los 200 días del año lectivo al menos una comida diaria a los 36 millones de niños de la enseñanza 
primaria y preescolar del sistema público y de establecimientos privados de naturaleza filantrópica. La población atendida supera en número a la población total de Canadá y es igual a la suma de las poblaciones de Australia, Grecia, Nueva Zelandia y Singapur. La cobertura del programa es prácticamente universal: en 1999 funcionaba en $96.7 \%$ de las escuelas públicas urbanas y en $98.1 \%$ de las rurales. Buena parte de los directores de escuela (75\% de los de escuelas rurales y $61 \%$ de los de las urbanas) cree que la merienda escolar es la comida principal del día para la mayoría de los alumnos.

El programa se ha mejorado, expandido y descentralizado totalmente. El gobierno actual duplicó casi las inversiones en merienda: desde 1995 hasta fines de 1999 el país invirtió 3600 millones de reales en la alimentación de los alumnos de la enseñanza primaria. Sólo en 1999 se destinaron 903 millones de reales, que representaron $33 \%$ de la dotación presupuestaria autorizada del Fondo Nacional de Desarrollo de la Educación (FNDE). Los recursos son enviados directamente a más de 5000 municipios, donde la comunidad y las escuelas deciden el menú con autonomía de acuerdo con los hábitos de alimentación locales y regionales y se encargan de controlar la calidad de los alimentos. A partir de este año, la constitución de un consejo municipal de merienda escolar pasó a ser una condición indispensable para que los recursos del programa sean traspasados a los municipios.

El antiguo Programa Nacional del Libro Didáctico (PNLD), a través del cual el gobierno federal adquiría y distribuía libros de texto de la enseñanza primaria, fue perfeccionado y ampliado a partir de 1995. Restringido a los primeros cuatro grados, con problemas crónicos de impuntualidad en la distribución y carente de una evaluación de contenidos y metodologías orientada por referentes curriculares nacionales adecuados, el antiguo programa dejaba mucho que desear.

A partir de 1996 el PNLD comenzó a ocuparse de todos los alumnos de primero a octavo grado de la educación primaria. Entre 1994 y 2000 fueron adquiridos directamente por el FNDE o en forma descentralizada por los estados de São Paulo y Minas Gerais, 597 millones de libros didácticos, escogidos por los propios profesores a partir de un catálogo de obras evaluadas por una comisión independiente de profesores. En 1999 se enviaron 502 títulos diferentes de 27 editoriales a 170 mil escuelas en todo el país.

Una sociedad entre el Ministerio de Educación y la Empresa Brasileña de Correos y Telégrafos garan- tiza la entrega del $100 \%$ de los libros en las escuelas antes del inicio de clases, incluso en los municipios más distantes. El programa está diseñado para que el libro sea reutilizado por un período de tres años, por lo cual se exigió a las editoriales mejorar la calidad del papel y las tapas, y se lanzó una campaña educativa dirigida a los alumnos y sus familias para estimular los cuidados en la conservación del material; la tasa de retorno ha sido sorprendentemente elevada. Según una evaluación independiente, $93.5 \%$ de los directores de escuela opinaron que los alumnos "utilizan con agrado" y aprueban los libros didácticos adoptados.

Para asegurar la permanencia en la escuela de los niños de menores ingresos, la iniciativa más importante y eficaz es sin duda el Programa Nacional de Garantía de Renta Mínima (PGRM), conocido como "Todo niño en la escuela". Dentro de la tendencia mundial a descentralizar los recursos y focalizar la atención, el programa otorga una ayuda financiera mensual a familias carentes (cuyo ingreso familiar es inferior a medio salario mínimo per cápita) con niños en edad escolar. El PGRM es instituido por los municipios en los que el ingreso familiar medio es inferior al promedio de su estado, pero aquellos que no estén en condiciones de sufragar el costo total del programa pueden recibir apoyo financiero de la Unión. En 2002 se pretende cubrir el universo de más de tres mil municipios aptos para participar del beneficio. A fines de 1999 el programa ya había favorecido a más de 500 mil familias, cerca de un millón de niños de 7 a 14 años, en más de mil municipios. El programa colabora también para erradicar el trabajo infantil y reducir el éxodo de las familias hacia las grandes ciudades.

El reconocimiento de la situación extremadamente desfavorable de la educación en la región Nordeste del país, que presenta indicadores muy inferiores a los promedios brasileños, condujo a establecer el Proyecto Nordeste en esa región. Además de aplicar cuantiosos recursos a programas municipales y estaduales de educación, el proyecto apoya las investigaciones que faciliten la formulación de estrategias eficaces para superar las deficiencias educacionales.

Entre 1993 y 1998, el Proyecto Nordeste invirtió recursos nacionales y externos - provenientes estos últimos de un préstamo del Banco Mundial- por cerca de 500 millones de dólares. Fue reformulado en 1998 con el nuevo nombre de Fondo de Fortalecimiento de la Escuela (Fundescola) y pasó a incluir también las regiones Norte y Centro-oeste.

El crecimiento cuantitativo y cualitativo de los indicadores educacionales en las regiones Nordeste y 
Norte ha superado con creces la media nacional. Esta evolución favorable, que se acentuó en los últimos años, muestra que las diferencias regionales se están reduciendo rápidamente. La educación primaria en ellas creció $27.2 \%$ a partir de 1994 , mientras que en el país en su conjunto creció $13 \%$; la matrícula de quinto a octavo grado subió $49 \%$, en tanto que la cifra a nivel nacional fue $27 \%$. Más de la mitad de los alumnos que cursan las clases de aceleración del aprendizaje están en la región Nordeste $(50.6 \%)$. En la enseñanza media, mientras la matrícula se expandió en $5.4 \%$, en la media del país entre 1999 y 2000 su crecimiento fue de $11 \%$ en el Nordeste y de $8.3 \%$ en el Norte.

\section{Educación infantil (de cero a seis años) y edu- cación especial}

En la educación infantil se destacan dos avances: la concepción de la atención educacional de niños de cero a seis años como una primera etapa de la educación básica, y la expansión de la matrícula. La inclusión de las guarderías infantiles (cero a tres años) en los sistemas de enseñanza y la definición de la responsabilidad de los municipios por la educación infantil completan los cambios institucionales efectuados. La matrícula preescolar, orientada a la atención de niños de cuatro a seis años, se expandió de $35.4 \%$ de esta población etaria en 1990 a $50.4 \%$ en 1998 . Otro dato positivo es que en el nivel preescolar, si bien los índices de cobertura siguen siendo insatisfactorios, las disparidades regionales son mucho menores que en los demás niveles de enseñanza y la distribución de las matrículas entre la zona rural y la urbana es mejor.

Mejorar el catastro de escuelas de educación infantil, sobre todo de aquellas que poseen guarderías, es una tarea que exige una gran movilización, ya que muchas funcionan informalmente ligadas a iglesias, asociaciones y organizaciones no gubernamentales. A fin de incluir toda esa red informal de atención en las estadísticas del Ministerio de Educación, el Instituto Nacional de Estudios e Investigaciones Educacionales (INEP) realizará a partir de octubre de 2000 el primer censo de educación infantil, cuyas informaciones serán fundamentales en la definición de políticas educacionales para el sector. Actualmente, el censo escolar que divulga anualmente el INEP recopila los datos de las guarderías y preescuelas. La realización de un censo exclusivo de la educación infantil tiene por objeto ampliar la cobertura de las informaciones recogidas.
En la educación especial, el análisis de la evolución habida en los años noventa muestra que el acceso a la educación de los portadores de necesidades especiales ha experimentado un notorio aumento. Este resultado es fruto del esfuerzo por superar tanto el paradigma como la práctica de la segregación y avanzar en una educación "inclusiva" en la red regular de enseñanza, en la cual el respeto a la diversidad de los alumnos sea el eje principal de la acción pedagógica. Entre 1988 y 1998 la matrícula de alumnos con necesidades especiales registró un crecimiento de $102.8 \%$.

\section{Alfabetización y educación de jóvenes adul- tos}

El crecimiento continuo de la tasa de escolaridad en todo el país viene reduciendo el analfabetismo, elevando el nivel de instrucción de la población y disminuyendo gradualmente las acentuadas disparidades regionales. El porcentaje de niños de 7 a 14 años de edad fuera de la escuela, que en 1989 se situaba en $16.2 \%$, disminuyó de $9.8 \%$ a $4.3 \%$ entre 1995 y 1999 .

En el grupo de 10 a 14 años de edad, en que se espera que el niño esté por lo menos alfabetizado, la tasa de analfabetismo bajó de $14.8 \%$ en 1989 a $9.9 \%$ en 1995 y cuatro años después cayó a 5.5\%. Pero la pesada herencia de analfabetismo y baja escolaridad media de la población, resultante de históricas deficiencias cuantitativas y cualitativas de la enseñanza primaria, obliga a seguir enfrentando estos flagelos con programas específicos.

Según dispone la Constitución, el Estado tiene el deber de promover la oferta gratuita de enseñanza para todos, incluso para aquellos que no tuvieron acceso a ella en la edad apropiada. Por lo tanto, se han puesto en marcha diversas experiencias de alfabetización de adultos y tentativas de reinserción tardía en la enseñanza primaria regular de jóvenes que habían abandonado la escuela.

Sin embargo, comenzó a difundirse cada vez más la noción de que la educación de jóvenes y adultos (EJA) requería una política específica, además de los contenidos y metodologías adecuados al universo del joven y el trabajador adulto. A partir de 1994, el gobierno federal comenzó a invertir en soluciones de esa índole, con programas orientados a la producción de materiales didácticos apropiados y la capacitación de profesores especializados. Se valorizaron sobre todo experiencias de asociación y otras experiencias exitosas realizadas por el sector empresarial, por organizaciones de la 
sociedad civil y por universidades y secretarías estaduales y municipales de educación.

Como resultado de esa política, el censo escolar de 1998 mostraba ya 2.8 millones de alumnos matriculados en cursos presenciales de educación de jóvenes y adultos, la gran mayoría de los cuales estaba terminando la enseñanza primaria.

En 2000 la EJA llegó a 3.1 millones de alumnos, considerando todos sus niveles. Siguiendo las mismas tendencias de la enseñanza regular, hubo un pequeño aumento en los grupos de alfabetización (3.7\%) y de primero a cuarto grado (2.3\%), y una expansión significativa en las clases de quinto a octavo grado (9.9\%). La enseñanza media se expandió notablemente: creció $33.3 \%$ respecto del año anterior, y abarcó 807600 estudiantes. En relación con el censo de 1995 ese crecimiento fue de $169.6 \%$.

Para combatir el analfabetismo en el grupo de 12 a 18 años - problema que se concentra en los municipios más pobres del país - se destaca la iniciativa denominada Alfabetización Solidaria, un proyecto innovador del programa Comunidad Solidaria, vinculado a la Presidencia de la República. Creado en enero de 1997, el proyecto, que opera en asociación con universidades y con iniciativas privadas, contabilizaba hasta diciembre de 1999 unos 300 mil alumnos en 866 municipios, la mayoría de las regiones Norte y Nordeste.

\section{Formación de profesores y calidad de la edu- cación}

Mejorar la calidad de la educación es la gran batalla que el Ministerio comenzó a librar en varios frentes. Se halla en curso una extensa reforma curricular de todos los niveles. Por primera vez en la historia de la educación brasileña el gobierno federal definió parámetros curriculares nacionales para los ocho grados de la enseñanza primaria, además de referentes para la educación infantil y reformuló todo el sistema de formación de profesores. Asimismo, se establecieron parámetros para la educación de jóvenes y adultos y para la educación indígena. Este último es uno de los aspectos más singulares de la política del actual gobierno, pues lo que se quiere es preservar y valorizar claramente la cultura de los grupos indígenas.

Uno de los rasgos más innovadores de los parámetros curriculares es su cobertura, que va mucho más allá de las materias que componen el currículo tradicional, pues tratan también temas vinculados con la formación del ciudadano y abordan cuestiones de éti- ca, ciudadanía y pluralidad cultural, además de educación ambiental, educación para la salud y orientación sexual.

Para la educación a distancia se crearon nuevos programas, como el de la televisión escolar (TV Escola), con el objetivo de ofrecer a sesenta mil escuelas en todo el país programas educativos de refuerzo escolar, asistencia a la labor del profesor y formación continuada de docentes. Transmitida en un canal exclusivo vía satélite, la televisión escolar proporciona tres horas de programación diaria de alta calidad, que se repite cuatro veces al día.

El Programa Nacional de Informática en Educación (PROINFO) demostró ser otra iniciativa importante. Ha capacitado hasta ahora a más de 20 mil profesores en el uso didáctico de la computadora. Hasta la fecha el gobierno ha instalado 30 mil computadoras y accesorios en más de dos mil escuelas de los 26 estados y del distrito federal, beneficiando directamente a cerca de 200 mil alumnos. La meta del programa es instalar de aquí a 2001 cien mil computadores en 6000 escuelas, lo que significa llegar a 7.5 millones de estudiantes. En 2000, gracias a la creación del Fondo de Universalización de los Servicios de Telecomunicaciones (FUST), el gobierno federal va a destinar 300 millones de reales a conectar las escuelas de la red pública a la Internet.

Para elevar la calidad de la enseñanza primaria es preciso hacer frente a otro gran desafío: la formación de profesores. Ese desafío lleva implícita la necesidad de valorizar el magisterio, mejorando las condiciones de trabajo y los salarios. El Fondo de Mantención y Desarrollo de la Educación Primaria y de Valorización del Magisterio (FUNDEF) comenzó a corregir el problema salarial, al destinar obligatoriamente $60 \%$ de sus recursos a la recalificación y remuneración de los profesores. Luego del primer año de funcionamiento del fondo, el salario medio nacional de los profesores aumentó en 13\%, pero el incremento llegó a $50 \%$ en las redes municipales del Nordeste, donde la remuneración era menor.

Además del aumento del número de profesores en los últimos cinco años $-10 \%$ en la enseñanza primaria y $36 \%$ en la enseñanza media- el censo escolar revela que ellos están más calificados, ya que ha mejorado considerablemente el nivel de su formación. Entre 1994 y 1999, la proporción de profesores sin formación adecuada - los llamados legos- cayó a 41\% en la enseñanza primaria, el número de profesores con educación media completa aumentó en $8 \%$ y el de aquellos con educación superior completa en $24 \%$. 
Entretanto, la Ley de Directrices y Bases de la Educación Nacional estipula que de aquí al año 2007 todos los profesores de la educación básica deben tener formación superior. En el país hay actualmente 600 mil profesores sin esa formación, de los cuales 95 mil no tienen siquiera la calificación mínima que se exige actualmente (habilitación de nivel medio para el magisterio). Casi $80 \%$ de los profesores de los primeros cuatro grados de la enseñanza primaria deberá ser recalificado, así como cerca de $25 \%$ de los docentes de los últimos cuatro grados.

Eso exigirá un gran esfuerzo conjunto de los tres niveles de gobierno en los próximos años, pues la tarea es gigantesca y el plazo es corto. Se están introduciendo grandes cambios en la organización del sistema de formación de profesores. La concepción de los institutos superiores de educación y de la carrera normal superior, la definición de programas especiales de formación pedagógica y la formulación de nuevas directrices curriculares para las carreras de pedagogía y para las licenciaturas en general, son medidas que deberán producir un fuerte impacto a corto plazo.

Entretanto, hay consenso entre los especialistas en educación de que ninguna formación inicial, ni siquiera la mejor de nivel superior, basta para el desarrollo profesional. Esa certeza hace indispensable la creación de un sistema de formación continuada y permanente para todos los profesores.

El gobierno viene invirtiendo cada vez más en cursos a distancia con el propósito de hacer viable la calificación de profesores en servicio. Algunos estados, con el apoyo de universidades, también desarrollan programas eficaces de calificación en servicio que llegan a millares de profesores en sus respectivas redes de enseñanza.

El programa de televisión escolar TV Escola, que se transmite por un canal exclusivo vía satélite, promueve la actualización del profesor mediante el apoyo sistemático a su labor en el aula. En la instalación del programa, el gobierno capacitó a 200 mil profesores y actualmente hay 56506 escuelas equipadas que reciben tres horas de programación diaria. En el aire desde hace tres años, la televisión escolar beneficia hoy a cerca de un millón de profesores y 28 millones de estudiantes.

\section{Educación media y técnico-profesional}

En la enseñanza media el aumento del número de matrículas fue vertiginoso: $11.5 \%$ en 1999 y $57 \%$ de 1994 a 1999. En la región Nordeste el crecimiento fue incluso mayor: $62 \%$.
La expansión impresionante de la matrícula en la enseñanza media se explica por tres factores principales: hay más estudiantes que están terminando la educación primaria; hay más alumnos que están egresando con menos edad y, por lo tanto, están en condiciones de continuar los estudios; y ha aumentado la demanda de los jóvenes de lograr una mayor escolaridad, incluso por las exigencias de un mercado laboral cada vez más competitivo. La enseñanza complementaria de nivel medio (educación de jóvenes y adultos a partir de los 18 años) creció $169 \%$ entre 1995 y 2000.

La educación media y la educación técnico-profesional están sufriendo una profunda reforma. Carente de identidad y desconectada de las exigencias del mundo moderno, la enseñanza media no estaba cumpliendo las funciones que se le exigían. Se le pedía que enseñara al estudiante a aprender, lo orientara en relación con la vida práctica y con una carrera profesional, y lo preparara para el ejercicio indispensable de la ciudadanía y la democracia. Además, debía estar en condiciones de enseñar al joven el uso de las nuevas tecnologías y formas de producir bienes, servicios y conocimiento.

El primer paso de la reforma de la educación de nivel medio fue de naturaleza estructural: se separó la educación media de la enseñanza técnico-profesional. Actualmente, una y otra existen en forma independiente y la segunda pasó a ser complementaria de la primera. Esta medida va a facilitar el logro de otra de las grandes metas del gobierno en pro de la educación, que es la cobertura también universal de la enseñanza media.

En la educación media, el Ministerio ha introducido, además de modificaciones estructurales, cambios de naturaleza didáctico-pedagógica y curricular. En el plano didáctico-pedagógico, la nueva enseñanza media va a asociar el conocimiento con la vida práctica del alumno, orientándolo respecto a su futuro y no siendo sólo una etapa de preparación para ingresar a la enseñanza superior.

Las directrices curriculares nacionales para la enseñanza media se definieron con carácter obligatorio para todas las escuelas del país. A continuación, el Ministerio elaboró los parámetros curriculares, conjunto de orientaciones y recomendaciones para apoyar la labor de los profesores en la nueva concepción de la enseñanza media.

Los currículos se tornaron más flexibles: $75 \%$ del contenido curricular se compone de una base nacional común y el $25 \%$ restante lo definen las propias escuelas basadas en las características socioeconómicas locales y regionales o en los intereses de la comunidad 
escolar. Con esa innovación el alumno pasa a tener mayor libertad para construir su propio currículo.

Junto con efectuar cambios en la educación media, el Ministerio dio inicio a la reforma de la educación técnico-profesional, según lo establece la Ley de Directrices y Bases de la Educación Nacional. Hoy, tal enseñanza se estructura en tres niveles independientes: básico (con independencia de cualquier escolaridad anterior), técnico (simultáneo o posterior a la enseñanza media) y tecnológico (de nivel superior, posterior a la enseñanza media). Las carreras técnico-profesionales de nivel medio, posmedio y superior se impartían tradicionalmente en escuelas presenciales con estructuras curriculares rígidas vinculadas a ocupaciones muy bien definidas y orientadas de preferencia al público joven.

Hoy, además de estar separada del ciclo medio, la educación técnico-profesional ofrece carreras que responden a las necesidades de los mercados de trabajo locales y regionales. El poder público propicia la diversificación de la enseñanza técnico-profesional de nivel posmedio, tanto técnico como superior, con amplia flexibilidad curricular y libertad para que jóvenes y adultos entren y salgan varias veces de los sistemas de enseñanza. La estructura curricular en módulos permite que el alumno siga varios cursos cortos en diferentes momentos de su vida y presenta contenidos flexibles que toman en cuenta las preferencias de los estudiantes. Con esta reestructuración, la educación técnico-profesional será capaz de habilitar de hecho al alumno para desempeñarse en diversas ocupaciones. Además, atraerá sólo a los jóvenes que realmente quieren trabajar como profesionales de nivel medio.

A través del Servicio Brasileño de Apoyo a la Micro y Pequeña Empresa (SEBRAE), 10 mil profesores de la enseñanza técnica están recibiendo capacitación para transmitir a sus alumnos nociones de administración y gestión empresarial, preparando a los estudiantes que así lo deseen para trabajar por cuenta propia o abrir una pequeña empresa al concluir su formación.

El Programa de Expansión de la educación técnico-profesional (PROEP), financiado por los Ministerios de Educación y de Trabajo y con apoyo del Banco Interamericano de Desarrollo (BID), está destinando 500 millones de dólares a reequipar escuelas técnicas públicas y a crear una red de escuelas técnico-profesionales comunitarias, en asociación con municipios, entidades sindicales y organizaciones civiles.

\section{Educación superior}

El sistema brasileño de educación superior, pese a ser reducido para las dimensiones del país y a presentar enormes diferencias de calidad, no llegó jamás a constituirse en un impedimento importante para el proceso de desarrollo brasileño.

El porcentaje de población con enseñanza superior completa entre los adultos mayores de 25 años de edad es semejante al de países como Austria, Chile, Italia y Uruguay, y superior al de China, India, Indonesia y Turquía.

En 1997 el 7\% de la población de 25 a 34 años contaba con enseñanza superior. En el grupo de 35 a 44 años el porcentaje aumentaba a $9 \%$. Y entre los adultos de 45 a 54 años y de 55 a 64 años, el porcentaje disminuía a $8 \%$ y $5 \%$, respectivamente. Los índices que registra Italia para cada uno de los grupos de edad señalados son parecidos: $8 \%$ (25 a 34 años), $11 \%$ (35 a 44 años), 8\% (45 a 54 años) y 5\% (55 a 64 años).

En la política de educación superior el desafío que debía enfrentarse - garantizar la expansión y diversificación del sistema con calidad - se delineaba claramente, dados el gran incremento de las tasas de conclusión de la enseñanza media y las exigencias de un mercado laboral cada vez más sofisticado y segmentado.

En términos cuantitativos, a partir de 1994 la expansión repuntó, después de un largo período de estancamiento del número de alumnos. La matrícula de la enseñanza superior creció en términos absolutos en los cuatro años siguientes más que en los 14 años anteriores, con 424 mil matrículas nuevas.

En 1998 había 2.1 millones de alumnos en la enseñanza superior, 28\% más que en 1994. El total de egresados representa hoy a $9 \%$ de la población con 21 años de edad. Este porcentaje es de 7\% entre los hombres y de $11 \%$ entre las mujeres.

En cuanto a la diversificación, se está reformando la estructura curricular de las carreras superiores que innova en dos aspectos: flexibiliza su estructura, lo que permite certificaciones parciales de carreras de corta duración, y abandona la fijación de currículos mínimos para cada carrera, sustituyéndola por el concepto de directrices curriculares por área de enseñanza.

En cuanto a la calidad, hasta 1995 la expansión del sistema contaba sólo con controles burocráticos $e x$ ante, sin que existiera un sistema de evaluación que permitiera vincular la acreditación de las instituciones con un juicio sobre su desempeño y calidad.

La legislación sobre la acreditación de cursos e instituciones se modificó sustancialmente, institucionalizándose la evaluación del desempeño como el mecanismo principal de acreditación y reacreditación. Se creó un innovador sistema de exámenes de finales de 
carrera a los que deben someterse los alumnos que egresan, como forma indirecta de evaluar el funcionamiento de los cursos respectivos. Los resultados de ese examen, sumados a la evaluación de las condiciones de funcionamiento efectuada por comisiones de especialistas designadas por el Ministerio, permiten que la sociedad sepa cuáles son las instituciones y las carreras con mejor desempeño. Además de democratizar la información, favorecer la fiscalización y el control social y señalar las deficiencias, esos resultados sirven de base para que el Ministerio decida si renueva o no la acreditación de instituciones y carreras.

A partir de la creación de ese sistema de evaluación integral, se otorgó mayor libertad a la expansión del sector privado en la enseñanza superior, sujeto al cumplimiento de patrones de calidad bajo la supervisión y evaluación sistemática del Ministerio de Educación. Se verificó un movimiento significativo de expansión hacia el interior del país y de corrección de los desequilibrios regionales. Cerca de un tercio del crecimiento de la oferta de vacantes en el sector privado tuvo lugar en las regiones Norte, Nordeste y Centro-Oeste.

Dado que buena parte de la expansión de la oferta se da y se seguirá dando en el sector privado, el gobierno se ha preocupado de perfeccionar los mecanismos de apoyo para que alumnos provenientes de familias de bajos ingresos accedan a la educación superior.

El nuevo Programa de Financiamiento Estudiantil (FIES), creado en 1999, otorgó en su primer semestre de funcionamiento créditos a más de 80 mil alumnos matriculados en facultades de todo el país. Dicha cifra supera en $173 \%$ la del último proceso selectivo realizado en 1997 por el antiguo sistema de crédito educativo y los recursos empleados sobrepasaron los 150 millones de reales en 1999.

En la educación superior pública, que es gratuita, se estimuló el aumento de la productividad del sistema, que presentaba una de las relaciones alumno/profesor más bajas del mundo. En el actual gobierno los recursos destinados por el sistema público federal a la enseñanza superior aumentaron $28 \%$ y se buscó transparencia y eficiencia en el uso de los mismos. Los recursos federales se distribuyen hoy a las universidades públicas según el número de alumnos y de egresados y se ha implantado un sistema de remuneración de los docentes vinculado con su desempeño académico.

Por consiguiente, la expansión de la enseñanza superior incorporó una característica nueva e impor- tante: la red pública retomó su dinamismo. El número de estudiantes de pregrado creció $17 \%$ de 1994 a 1999 y el de aquellos de posgrado registró avances significativos: entre 1995 y 1998 los alumnos de maestría subieron de 43 mil a 51 mil y los de doctorado de 16 mil a 27 mil. Esto significa que Brasil forma 13 mil maestros y 4 mil doctores por año.

Se está invirtiendo en recursos humanos y en equipos e instalaciones físicas. Mejoró la calificación media del cuerpo docente, pues la proporción de doctores subió de $22 \%$ a $29 \%$. Se dio prioridad a la enseñanza de pregrado, dedicando más de cien millones de reales a bibliotecas, computadoras e infraestructura de informatización. Se realizó una licitación internacional para adquirir equipos de laboratorio destinados a la enseñanza de pregrado y a hospitales universitarios por un valor de 300 millones de dólares, los que ya se están entregando a las instituciones públicas de enseñanza superior.

\section{Información y evaluación}

En este gobierno hubo una verdadera revolución en términos de información y evaluación educacionales. La alta calidad de las investigaciones las ha convertido en un instrumento indispensable para la planificación y ejecución de las políticas públicas del Ministerio de Educación. Gracias a ellas el gobierno, además de gastar más en educación, está gastando mejor los recursos.

El punto de partida fue la transformación del Instituto Nacional de Estudios e Investigaciones Educacionales (INEP) en una entidad autónoma (autarquía). En esa condición, el INEP pasó a encargarse de todo el sistema de captación, evaluación y almacenamiento de la información sobre todo el ámbito de la educación en el país.

El Sistema de Evaluación de la Enseñanza Básica (SAEB), que investiga el desempeño de los alumnos de enseñanza primaria y media, es reconocido como uno de los más sofisticados del mundo en materia de evaluación del rendimiento escolar.

El Examen Nacional de Enseñanza Media (ENEM), que se realizó por tercera vez en 2000, tiende a afianzarse como un instrumento importante de evaluación del desempeño de los alumnos y las escuelas. Este año, 350 mil estudiantes rindieron el examen que ya es aceptado por 130 instituciones como prueba válida para acceder a la enseñanza superior, en forma aislada o combinado con el examen de ingreso tradicional. 
A partir del año que viene las inscripciones para este examen serán gratuitas para todos los alumnos de las escuelas públicas. El objetivo es presionar a las universidades federales para que acepten el examen: entre las más de 50 universidades federales brasileñas, sólo siete lo adoptan actualmente como una de las formas de ingreso. Se espera que de un universo de 1.8 millones de alumnos que egresarán de la enseñanza media en 2001, habrá unos 900 mil candidatos que se inscribirán en el ENEM.

En la educación superior se ha consolidado el Examen Nacional de Cursos (Provão) tras cuatro años de aplicación; cubre actualmente 18 carreras superiores y evalúa 2889 cursos mediante un examen que se toma a nivel nacional a 214 mil alumnos (70\% del total de alumnos de pregrado). El examen nacional, que es esencial para evaluar la calidad de las carreras superiores de pregrado, está contribuyendo notablemente a mejorar el nivel de las instituciones educativas.

La calidad de la labor realizada por el INEP en los últimos cinco años ha conquistado el respeto internacional. Gracias a los avances logrados en los sistemas de evaluación e información, el país participa hoy en estudios internacionales comparativos como el World Educational Indicators (WEI) de Unesco/OCDE y el PISA 2000 de la Organización de Cooperación y Desarrollo Económicos (OCDE), que permiten un diagnóstico preciso de la situación de Brasil en relación con los demás países.

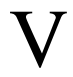

\section{Los recursos públicos para la educación}

La legislación brasileña asegura un flujo regular de recursos públicos a la educación. El gasto público de Brasil en educación, comparado con el producto interno bruto, es uno de los mayores entre los países que participan en el proyecto WEI y es igual a la media de los países de la OCDE.

En 1997 el gasto público en educación ascendía a 37 mil millones de reales. Equivalía a $4.8 \%$ del PIB, cifra mayor que la de Argentina (3.7\%), Chile (3.2\%), Filipinas (3.0\%), México (4.6\%), Malasia (4.4\%), Tailandia $(4.3 \%)$ y Uruguay $(2.6 \%)$ y semejante a la de España (4.8\%) y a la media de los países de la OCDE (4.9\%).

En 1997 el gasto total fue de 43.3 mil millones de reales, desglosado en 8.6 mil millones de la Unión (19.8\%), 21 mil millones de los estados (49.8\%) y 13.1 mil millones de los municipios (30.4\%). El gobierno federal aporta $62 \%$ de los recursos invertidos en la educación superior y ejerce una acción subsidiaria considerable en la educación primaria y media.

En el mismo año el gasto en personal y cargas absorbía $57.6 \%$ de los recursos directos aportados por las tres esferas gubernamentales. Esa participación tiende a aumentar gracias a las políticas de capacitación y valorización del magisterio. Entre 1994 y 1999 hubo un crecimiento de $45.3 \%$ del número de profesores con formación superior completa y una caída de $65.8 \%$ del número de profesores legos (sin habilitación de nivel secundario).
La Constitución de 1988 establece que los estados y municipios están obligados a destinar a la educación al menos $25 \%$ de los ingresos resultantes de la recaudación tributaria, y dedicar al menos $60 \%$ de esos recursos a la enseñanza primaria. En el caso de la Unión, el porcentaje mínimo es de $18 \%$ de la recaudación.

Además, la enseñanza primaria cuenta con recursos complementarios que provienen del "salario-educación", una contribución social exigida a las empresas por un valor equivalente a $2.5 \%$ de la nómina. De esos recursos, un tercio se destina a un fondo federal, el FNDE, que elabora programas de apoyo destinados exclusivamente a las redes estaduales y municipales de enseñanza primaria, y dos tercios se destinan directamente a los estados donde se verifica la recaudación. En 1998 los recursos destinados por las tres esferas de gobierno exclusivamente a la educación primaria totalizaron 18.3 mil millones de reales.

Si no fuera por la mala distribución de las partidas existentes, sumada a su mala aplicación, los recursos disponibles serían suficientes para mantener un sistema educacional de mucho mejor calidad y mayor cobertura que el que efectivamente existía en el país hasta 1995.

La mala distribución emanaba de las grandes diferencias de capacidad de recaudación entre los esta- 
dos y municipios más ricos y los más pobres. Los primeros no destinaban el $25 \%$ de su recaudación a la educación primaria obligatoria o a la educación infantil: invertían una parte importante de esos recursos en la enseñanza media y, en algunos casos, incluso en la superior. En los municipios más pobres, especialmente en la región Nordeste, los recursos no eran suficientes para asegurar una educación de calidad. En muchas de esas localidades el gasto medio por alumno/año era inferior a 100 reales y el salario de los profesores no llegaba ni siquiera al salario mínimo vigente. Por lo tanto, no había una relación entre los recursos disponibles y el número de alumnos atendidos por las diferentes redes de enseñanza.
La mala aplicación iba desde la destinación de los recursos de la educación primaria a otros niveles de enseñanza hasta el desvío puro y simple de las partidas, que se perdían en los meandros de la burocracia antes de llegar a la escuela, tanto por falta de una definición clara de lo que podía incluirse como gasto en educación como por falta de control y fiscalización.

Precisamente para corregir tales distorsiones y poner en práctica las propuestas de la nueva Ley de Directrices y Bases de la Educación Nacional el actual gobierno propuso y aprobó en 1996 la enmienda constitucional número 14 que creó un Fondo de mantención y desarrollo de la educación primaria y de valorización del magisterio (FUNDEF).

\section{VI}

\section{EI Fondo de mantención y desarrollo de la educación primaria y de valorización del magisterio (FUNDEF)}

La enmienda constitucional número 14 que estableció el FUNDEF, estipula que durante un decenio, a partir de su promulgación, los estados y municipios deberán destinar a la educación primaria no menos de $60 \%$ de los recursos que ya estaban vinculados con la educación en virtud de la Constitución de 1988. Aunque el FUNDEF no recoge todos los impuestos recaudados por estados y municipios, esa obligatoriedad se aplica a todos los ingresos que componen los presupuestos de esas esferas de gobierno.

Los recursos destinados previamente "a la educación" en forma genérica - $y$ sin que estuviera definido claramente lo que podía considerarse gasto en educación - no se asignaban necesariamente a las prioridades reales definidas por el país: la enseñanza primaria, el incremento del número de alumnos, una mejor remuneración del profesor, un nivel de recursos que rescate la calidad de las escuelas de las regiones más pobres.

Implantado en 1998 en todas las unidades de la federación por un plazo de diez años, el FUNDEF es un fondo contable (es decir, no aporta nuevos recursos sino que distribuye en forma diferente los de fuentes ya disponibles) que abarca a las principales fuentes de ingreso estaduales y municipales.
Antes de la creación de este mecanismo, la repartición de los ingresos tributarios entre estados y municipios no guardaba ninguna correspondencia con la división de cargas educacionales entre las redes estaduales y municipales de enseñanza. Eso acentuaba sobremanera las desigualdades entre los municipios más ricos y más pobres dentro de cada estado, además de no ayudar a combatir los tradicionales desequilibrios regionales del país.

Después de la creación del FUNDEF, la redistribución entre cada estado y sus municipios de los recursos que componen el fondo se realiza automáticamente, de acuerdo con el número de alumnos matriculados en las respectivas redes de educación primaria.

Con la nueva definición - ahora con fuerza de ley- de lo que puede incluirse como gasto en educación, los valores redistribuidos por el fondo se depositan en una cuenta bancaria específica, lo que mejora notoriamente la fiscalización pública de su utilización.

Los recursos recibidos por las redes estaduales o municipales de enseñanza en la cuenta del fondo deben asignarse de modo de satisfacer las siguientes exigencias:

i) un mínimo de $60 \%$ debe destinarse a la remuneración de los profesores que ejercen efectivamente 
en la educación primaria, permitiéndose entretanto la utilización de parte de esos recursos en la capacitación de profesores legos hasta 2001;

ii) el $40 \%$ restante debe dedicarse a acciones definidas por ley como de mantenimiento y desarrollo de la educación: construcción, ampliación, terminación o reforma de escuelas, adquisición de material didáctico y equipos, servicios diversos y pago de jubilaciones.

El gobierno federal garantiza una "complementación" cuando la distribución del fondo en cada estado no alcanza un cierto mínimo nacional por alumno que se estipula anualmente y que hoy es de 333 reales por alumno/año en los primeros cuatro grados de la enseñanza primaria y de 349.65 reales en los últimos cuatro.

En 1998, primer año de funcionamiento del fondo, se aseguró la destinación de casi 13.3 mil millones de reales a la enseñanza primaria obligatoria. En 2000 el ingreso previsto del fondo es de 17 mil millones de reales. La región Sur participará con 2.5 mil millones de reales, el Sudeste con 8 mil millones, el Centro-oeste con 1.1 mil millones, el Nordeste con 4 mil millones y el Norte con 1.4 mil millones. Los estados más pobres, que no alcanzan al gasto mínimo por alumno, recibirán recursos complementarios: 511.6 millones de reales para el Nordeste y 122.6 millones para el Norte. En las demás regiones, los estados superan el gasto mínimo por alumno.
La enmienda constitucional que creó el fondo exigió también la creación de consejos integrados por representantes de la sociedad civil en las diversas instancias del gobierno con el fin de fiscalizar los gastos efectuados.

Ultimamente, ante denuncias de irregularidades en la asignación de los recursos del FUNDEF —no asignación de $60 \%$ de los recursos a la enseñanza primaria; financiamiento de actividades no consideradas como de mantenimiento y desarrollo de la enseñanza- en cerca de $5.5 \%$ de los municipios, el gobierno determinó con carácter permanente el término del secreto bancario de las cuentas de todas las prefecturas y gobiernos estaduales donde se depositan los recursos de la educación. Además, entabló demandas en los tribunales de cuentas y el ministerio público de los estados donde ocurrieron los hechos, lo que ya derivó en el despido de cinco prefectos y en más de 100 sumarios en curso en 20 estados.

Los diputados estaduales, ediles, miembros del ministerio público y de los tribunales de cuentas de los estados y municipios, además de los integrantes de los consejos de fiscalización del fondo, tienen hoy libre acceso a los extractos de las cuentas del fondo. Por lo tanto, el FUNDEF constituye un ejemplo innovador de una política social transparente que articula los tres niveles de gobierno e incentiva la participación de la sociedad en la fiscalización de la gestión de los recursos.

\section{VII}

\section{Otras fuentes de financiamiento}

\section{Recursos internacionales}

Los empréstitos externos son importantes para llevar a cabo proyectos especiales, siempre que el financiamiento ordinario del sistema no dependa de esos recursos. En esos términos, la cooperación internacional ha sido muy relevante en Brasil, bajo la forma de asistencia técnica y financiamiento de investigaciones y proyectos. Tanto el Banco Interamericano de Desarrollo (BID) como el Banco Mundial constituyen fuentes importantes para financiar proyectos que tienen como objetivo central mejorar la calidad de la educación y la equidad del sistema educacional.

La iniciativa principal favorecida con recursos del Banco Mundial es el Proyecto Nordeste, orientado a las áreas más pobres del país, y que hoy reformulado y ampliado bajo el nombre de FUNDESCOLA atiende también las regiones Norte y Centro-oeste del país. El BID apoya el importante Programa de Expansión de la Educación Profesional (PROEP), por un valor total de 500 millones de dólares, que incluye recursos de los Ministerios de Educación y Trabajo; además, contribuye en programas como la Red Internacional Virtual de Educación (RIVED), que reúne a especialistas de Brasil, Venezuela y Colombia para promover el desarrollo de programas computacionales de apoyo a la enseñanza de matemáticas y ciencias en las escuelas públicas.

Sumándose a los recursos internos, incluidos los estaduales, el BID financiará también la mitad del total 
de 500 millones de dólares que se destinarán al innovador proyecto PROMED-Escuela Joven. El objetivo es dotar a las escuelas de las condiciones necesarias para la aplicación de la reforma curricular de la enseñanza media. El PROMED se orienta sobre todo a la creación de espacios escolares destinados específicamente a los jóvenes, mediante la construcción, ampliación y mejoramiento de la red física y la instalación de bibliotecas, laboratorios para la enseñanza de ciencias y equipos de informática. Por otra parte, el programa estimulará el protagonismo juvenil, previendo la incorporación de proyectos que serán desarrollados por los alumnos en cualquier área — ciencias, artes, deportes, iniciativas comunitarias - de las actividades regulares de las escuelas.

\section{Asociaciones con la comunidad}

El objetivo principal del vasto programa de asociación con la comunidad desarrollado por el Ministerio de Educación -Acorda, Brasil. Está na hora da Escola! - es sembrar en el país una cultura de compromiso con la educación, sobre todo con la primaria. Desde la dueña de casa que transforma su residencia en lugar de matrícula hasta el padre del alumno que pinta o repara las instalaciones de la escuela de su hijo, desde el artista consagrado que participa gratuitamente en las campañas de escolaridad hasta el profesor jubilado que dicta clases de recuperación ad honorem, hay millares de acciones cuyo valor es imposible medir en términos monetarios.

Pero, además de incentivar la colaboración espontánea entre el estado y la comunidad para resol- ver los problemas educacionales, el programa busca también estructurar asociaciones formales con los agentes sociales. Hasta septiembre de 1998 había estructurado 121 sociedades, que significaron inversiones por casi 25 millones de reales destinadas a proyectos educacionales, donaciones de equipo y material didáctico-pedagógico, cesión de espacios publicitarios y capacitación de profesores, que beneficiaron a alrededor de 20 millones de alumnos de la red pública en todo Brasil.

A medida que la comunidad va adoptando paulatinamente una postura más proactiva con relación a la escuela crece el número de empresas, órganos de comunicación, fundaciones y organizaciones no gubernamentales, gobiernos estaduales y municipales, institutos y asociaciones empresariales e instituciones financieras que están colaborando con la educación, incluso sin ser formalmente "socios" del gobierno. Por ejemplo, una empresa financiera por sí sola mantiene 36 escuelas en 23 estados y ofrece enseñanza preescolar, primaria, media, complementaria y técnico-profesional a cerca de 95 mil estudiantes, con una inversión de 80 millones de reales al año.

Las donaciones de laboratorios de informática, equipos de televisión, videos educativos, enciclopedias, concursos y premios, las actividades deportivas y artísticas fuera de clases, la participación en asociaciones de padres y en consejos escolares, la fiscalización de los programas y la asignación de las partidas públicas son formas de participación que van en aumento y contribuyen a crear el capital social que es la condición y consecuencia de la revolución silenciosa que Brasil está realizando en la educación.

\section{VIII}

\section{Desafíos y perspectivas}

El gran desafío actual para la educación brasileña ya no es la cobertura universal de la enseñanza primaria y ni siquiera la erradicación del analfabetismo, sino la búsqueda de índices crecientes de calidad en todos los niveles, lo que está íntimamente vinculado con la calificación del cuerpo docente.

Entre 1991 y 1998 disminuyó el número absoluto de personas analfabetas, de 19.2 millones a 15.2 millones, y hubo una rápida disminución de las tasas de analfabetismo en los grupos más jóvenes beneficia- dos por la mayor cobertura del sistema escolar. Brasil logró dar acceso universal a la educación primaria, amplió la cobertura de la enseñanza media y superior y mejoró la calificación de los profesores.

Pero es preciso hacer mucho más: aplicar la reforma curricular, aumentar el número de docentes con educación superior, ampliar con calidad las matrículas en la enseñanza media y aumentar los años de escolaridad de la población. Actualmente, ya no basta con garantizar la cobertura universal de la enseñanza 
primaria de ocho años. Para tener una ciudadanía plena y llevar una vida productiva es imprescindible tener como mínimo 12 años de escolaridad.

La rapidez con que Brasil ha logrado ampliar la cobertura de la enseñanza secundaria tal vez no tenga paralelo en ningún otro país. Pero la tasa neta de escolarización (33.4\% de la población de 15 a 17 años) todavía se considera baja, pese a haber crecido $57 \%$ entre 1994 y 1999.

El desafío de formar y actualizar profesores para la nueva etapa de ampliación de la enseñanza media y técnico-profesional es inmenso. Habrá que echar mano a las nuevas tecnologías, con una creciente combinación de educación presencial y educación a distancia. Será preciso desarrollar programas computacionales, programas de televisión, módulos interactivos de aprendizaje por la Internet, nuevas tecnologías donde el profesor actúa en el aula como un monitor polivalente que utiliza los recursos de la enseñanza a distancia y a la vez aprende y se actualiza.

Las universidades se están preparando para asumir el liderazgo en este proceso, integrándose en redes para la enseñanza virtual. La Uni-Red es un consorcio que fue creado en enero de 2000 y está integrado por 65 universidades públicas, federales y estaduales que cubren todo el territorio nacional por la Internet y la televisión por cable, para atender en conjunto la demanda de enseñanza pública superior en todo el país. Cada institución va a producir en las áreas donde es más competente y esos programas van a transmitirse y compartirse. El primer programa ofrecerá licenciaturas en áreas definidas de acuerdo con las necesidades nacionales, regionales y locales. La formación de profesores en servicio para atender las exigencias de formación superior establecidas por la Ley de Directrices y Bases de la Educación Nacional es uno de los objetivos prioritarios de la Uni-Red, que pretende atender a 100 mil alumnos/año, llevando contenidos y recursos pedagógicos elaborados por las mejores universidades brasileñas a los rincones más apartados del país.
No sólo en el campo de las licenciaturas, sino en todas las áreas de enseñanza superior, la tendencia a combinar la educación a distancia y la enseñanza presencial dentro de las instituciones se torna cada vez más fuerte. Las universidades van a poder tener acceso a cursos compactos de los mejores centros del mundo: al premio Nobel de macroeconomía, al mejor especialista en determinado tipo de cirugía, y en su cuerpo docente, al profesor preparado para trabajar con esa metodología y traspasarla a los estudiantes. No se trata de la antigua enseñanza a distancia, del alumno que se queda en casa, extrae de noche el material de la Internet y hace las tareas en su domicilio. Eso no es gran cosa; lo importante va a ser la combinación del trabajo presencial con los nuevos recursos de la tecnología.

Por cierto, el financiamiento de la educación es un problema en todo el mundo. El gasto público de Brasil en educación, como proporción del PIB, es uno de los más elevados entre los países que participan en el proyecto de Unesco/ocDE e igual a la media de los países de la OCDE. Los esfuerzos del gobierno en busca del equilibrio fiscal no han afectado en forma significativa las inversiones en educación. Por otra parte, se ha avanzado mucho en el mejoramiento de la productividad dentro del sistema, pero hoy los incrementos que se logran son ya marginales.

Brasil tendrá que seguir recurriendo al financiamiento internacional para asegurar, por una parte, la expansión y mejoramiento de la red física de establecimientos de enseñanza y, por otra, la utilización intensiva de las tecnologías de educación presencial y a distancia más avanzadas. Sería del todo deseable, no sólo para Brasil sino para todos los países prestatarios, que esos empréstitos no se calcularan conforme a los límites de endeudamiento incorporados en las metas de los programas de ajuste fiscal de las cuentas públicas.

Las perspectivas del sistema nacional de educación son muy promisorias. Se ha avanzado mucho, el país está mucho mejor de lo que estaba, pero aún queda un largo camino por recorrer.

(Traducido del portugués) 